\title{
MINIMALLY INVASIVE PORT-ACCESS MITRAL VALVE SURGERY
}

F. W. Mohr, MD, PhD

V. Falk, MD

A. Diegeler, MD

T. Walther, MD

J. A. M. van Son, $\mathrm{MD}, \mathrm{PhD}$

R. Autschbach, MD, PhD

Sponsor:

Hans G. Borst, MD
Objectives: This study evaluates the feasibility of video-assisted minimally invasive mitral valve surgery by means of the Port-Access system. The aim of the study was to minimize surgical access and to develop a video-assisted surgical technique. Methods: The Port-Access system allows for closed chest endoluminal aortic clamping, cardioplegic arrest, and decompression of the heart. The mitral valve was either repaired $(n=28)$ or replaced $(n=23)$ in 51 patients by means of a minimally invasive approach through a right lateral minithoracotomy and under videoscopic guidance. Results: Mean length of incision was $5.4 \pm 1.8 \mathrm{~cm}$ (range 3.8 to $8 \mathrm{~cm}$ ). Mean duration of operation, cardiopulmonary bypass, and crossclamp time was $196 \pm 53$, $133 \pm 52$, and $72 \pm 27$ minutes, respectively. Median intubation time was $\mathbf{2 5 . 5}$ hours (range 5 to 264 hours). Median duration of intensive care and hospital stay was 2 days (range 1 to 36 days) and 13 days (10 to 36 days), respectively. Hospital mortality was $9.8 \%$ (5/51). Overall morbidity was relatively high. In two patients acute retrograde aortic dissection led to conversion of the procedure. At follow-up (261 \pm 13 days), three patients required reoperation for paravalvular leakage. Baseline mean Duke activity index score was $19.3 \pm 11.3$ before the operation and increased to $23.2 \pm 10$ at 6 weeks' and $24.2 \pm 10.3$ at 12 weeks' follow-up, respectively. Conclusion: The Port-Access system allows for video-assisted minimally invasive replacement and complex repair of the mitral valve through a right lateral minithoracotomy. However, morbidity and mortality associated with this novel technique were high. (J Thorac Cardiovasc Surg 1998;115:567-76)
$T_{\text {the }}^{\text {he }}$ he Port-Access endovascular cardiopulmonary bypass system (EndoCPB, Heartport, Inc., Redwood City, Calif.) is a closed chest endovascular system that enables aortic clamping, cardioplegic arrest, cardiac decompression, and venting of the left side of the heart. ${ }^{1-3}$ In animal models and cadaver studies this technique has been used successfully for minimally invasive coronary artery bypass grafting and mitral valve replacement. ${ }^{3,4}$ First clinical results have been recently reported. ${ }^{5,6}$ This

From the Department of Cardiac Surgery, Herzzentrum, Universität Leipzig, Leipzig, Germany.

Read at the Seventy-seventh Annual Meeting of The American Association for Thoracic Surgery, Washington, D.C., May 4-7, 1997.

Received for publication May 5, 1997; revisions requested June 17, 1997; revisions received Oct. 20, 1997; accepted for publication Oct. 21, 1997.

Address for reprints: F. W. Mohr, MD, PhD, Universität Leipzig, Herzzentrum, Klinik für Herzchirurgie, Russenstraße 19, D-04289, Leipzig, Germany.

Copyright (c) 1998 by Mosby, Inc.

0022-5223/98 $\$ 5.00+0 \quad \mathbf{1 2 / 6 / 8 7 0 4 2}$ report describes our early results with minimally invasive mitral valve surgery performed with the Port-Access system in 51 patients.

\section{Methods}

After approval by the local ethical committee of the University of Leipzig, 51 consecutive patients ( 17 men, 34 women, mean age $61.8 \pm 10.7$ years) with nonischemic mitral valve disease were prospectively included in the study from June 1996 to March 1997. All patients gave written informed consent after the study protocol and the potential risks associated with the procedure had been outlined in detail. Demographics and preoperative patient data are summarized in Table I.

Anesthesia. Patients were positioned in a supine position with the right side of the chest slightly elevated. Intravenous anesthesia with sufentanil, propofol, and pancuronium was administered. The airway was intubated with a left-sided double-lumen endobronchial tube (Mallinckrodt, Athlone, Ireland) in the first 10 patients, whereas later in the series single-lumen intubation was performed. An arterial pressure line was placed in the right radial artery. A 5F single-lumen flow-directed pulmonary artery catheter (Arrow International, Inc., Reading, Pa.) was inserted through a $9 \mathrm{~F}$ venous sheath in the right jugular vein and positioned in the pulmonary artery 


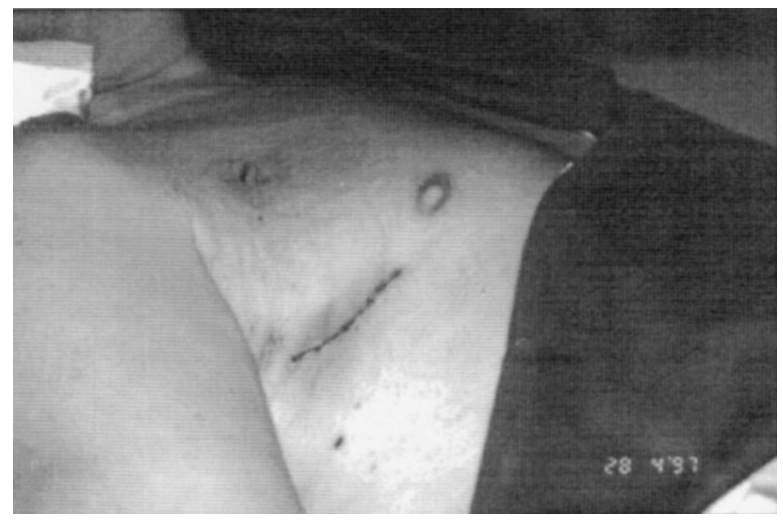

Fig. 1. Incision for minimally invasive Port-Access mitral valve surgery.

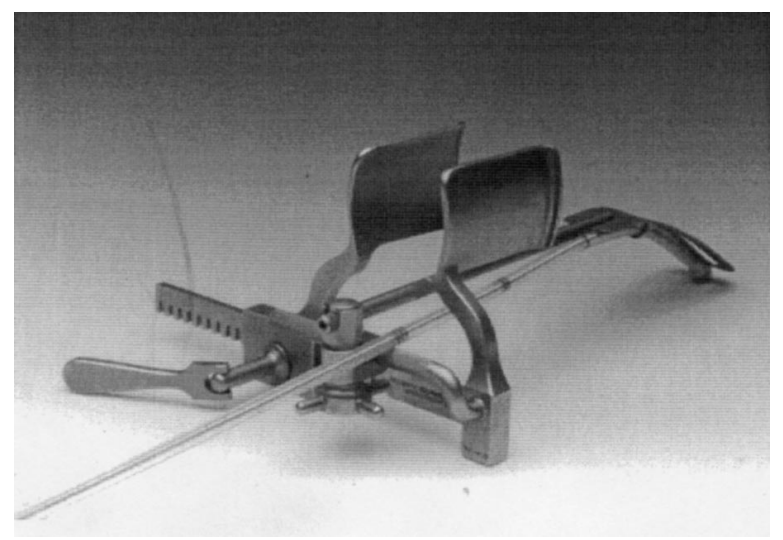

Fig. 2. Specially designed rib retractor with integrated arm to enhance exposure of the mitral valve and housing a left atrial vent.

under pressure control. A single-lumen $8.3 \mathrm{~F}$ endovascular pulmonary vent (Heartport) was then introduced and positioned over the flow-directed catheter in the main pulmonary artery. The vent was connected via an air relief valve to the cardiopulmonary bypass venting line.

Surgery. All patients were operated on at the Heartcenter of the University of Leipzig by one experienced surgeon. The right femoral artery and vein were surgically exposed through a 3 to $4 \mathrm{~cm}$ incision parallel to the inguinal skin fold. After systemic heparinization, a standard $28 \mathrm{~F}$ venous return cannula (DLP, Inc., Grand Rapids, Mich.) was placed in the femoral vein and advanced to the right atrium under transesophageal echocardiographic (TEE) control. A $21 \mathrm{~F}$ or $23 \mathrm{~F}$ Y-shaped arterial return cannula (Heartport) with one arm for arterial return and one arm for placement of the endovascular aortic clamp was placed in the femoral artery. A conventional cardiopulmonary bypass system with roller pumps and membrane oxygenator was used. In addition, a centrifugal pump (Medtronic Bio-Medicus, Eden Prairie, Minn.) was placed in the venous line to enhance venous drainage.
Table I. Patient characteristics and parameters of cardiac function

\begin{tabular}{lc}
\hline Patient characteristics & \\
Mean age (yr) & $68.1 \pm 10.7$ \\
Male/female $(n)$ & $17 / 34$ \\
Reduced vital capacity $(n)$ & $8(16 \%)$ \\
Hypertension $(n)$ & $20(39 \%)$ \\
Diabetes $(n)$ & $13(25 \%)$ \\
Preoperative NYHA functional class & \\
Class I $(n)$ & 2 \\
Class II $(n)$ & 11 \\
Class III $(n)$ & 38 \\
ASA class & \\
Class II $(n)$ & 14 \\
Class III $(n)$ & 32 \\
Class IV $(n)$ & 5 \\
Mitral valve and LV function & \\
Mitral insufficiency $>$ secondary $(n)$ & 39 \\
Mitral stenosis $>$ secondary $(n)$ & 12 \\
Mitral valve orifice area $\left.(\mathrm{cm})^{2}\right)$ & $2.8 \pm 1.5$ \\
$\Delta$ Pmax (mm Hg) & $7.8 \pm 6.8$ \\
$\Delta$ Pmean (mm Hg) & $3.2 \pm 3.8$ \\
Left ventricular end-diastolic diameter $(\mathrm{mm})$ & $50 \pm 13$ \\
Diameter of left atrium (mm) & $52 \pm 11$ \\
Left ventricular end-diastolic pressure $(\mathrm{mm} \mathrm{Hg})$ & $14 \pm 5$ \\
Ejection fraction $(\%)$ & $57 \pm 13$ \\
Fraction of shortening $(\%)$ & $27 \pm 10$ \\
Preoperative rhythm & \\
Sinus rhythm $(n)$ & 18 \\
Atrial fibrillation $(n)$ & 33 \\
Tricuspid regurgitation (mild to moderate) $(n)$ & 32 \\
\hline
\end{tabular}

NYHA, New York Heart Association; ASA, American Society of Anesthesiology; $L V$, left ventricular; $\triangle P \max$, maximum pressure change; $\Delta$ Pmean, mean pressure change.

Synchronous with exposure of the femoral vessels, a small horizontal incision was made laterally over the fourth intercostal space between the anterior and median axillary lines (Fig. 1). A specially designed rib retractor (Arnold Medizintechnik, Tuttlingen, Germany) was inserted (Fig. 2). After initiation of cardiopulmonary bypass the lungs were deflated. The pericardium was opened 3 $\mathrm{cm}$ above and parallel to the right phrenic nerve to expose the roof of the left atrium. Exposure was enhanced by placing three stay sutures in the pericardium, which were transcutaneously fixed outside the chest. A $10 \mathrm{~mm}$ port was inserted at the second intercostal space in the anterior axillary line to allow placement of a videoscope (Endolive 3D Video Thoracoscope System, Carl Zeiss, Oberkochem, Germany) into the left atrium. Cardiopulmonary bypass was instituted with the use of moderate hypothermia at $30^{\circ} \mathrm{C}$. Before insertion of the endovascular aortic clamp with the aid of TEE, the aorta was screened for atheromatous debris and thrombi to avoid cerebral embolization with retrograde perfusion. Advancement of the guide wire could be visualized from the descending aorta to the aortic valve by TEE. Correct placement of the clamp (1 cm above the level of the sinotubular junction) was controlled by fluoroscopy (Sieremobil 2000, Siemens, Erlangen, Germany) and multiplane TEE (Vingmed 800 

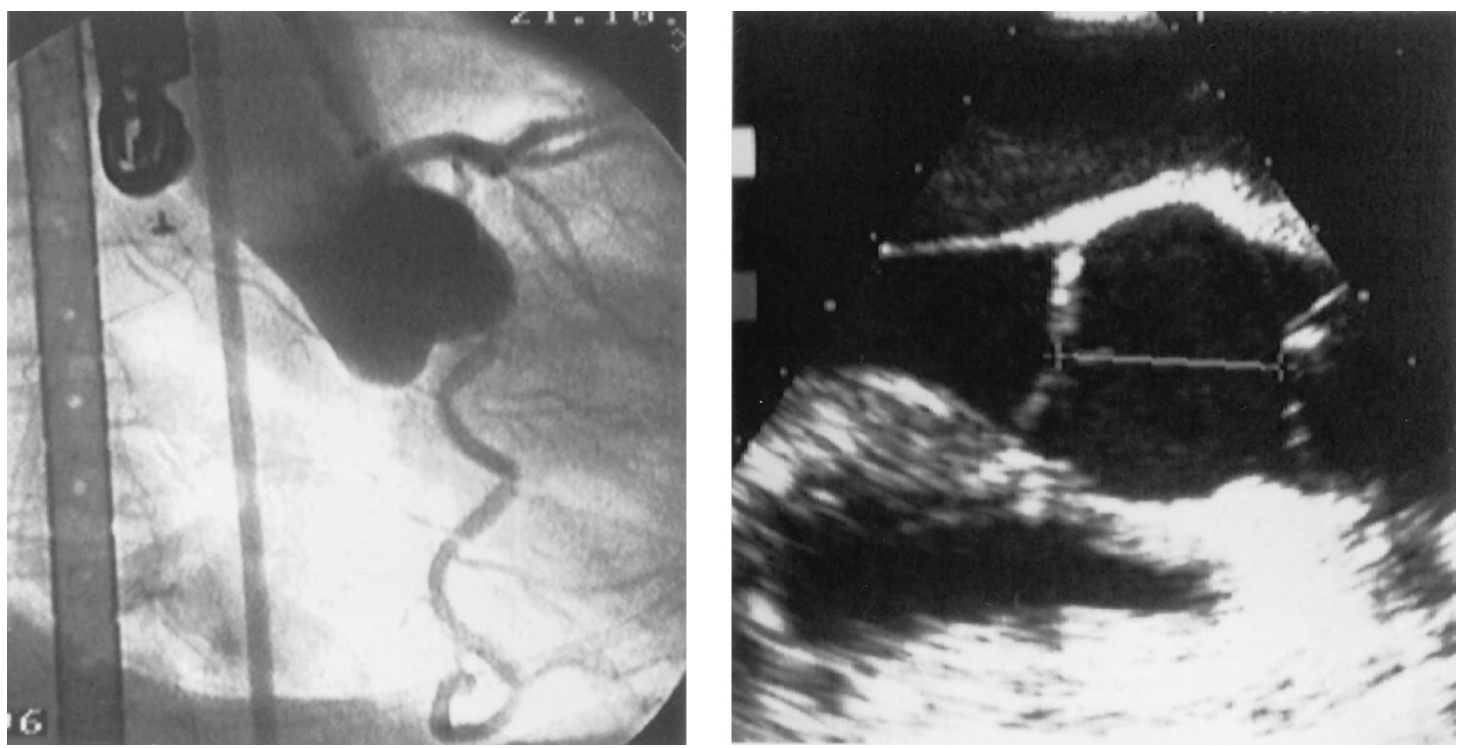

Fig. 3. Intraoperative fluoroscopy showing inflated endovascular aortic clamp positioned in the ascending aorta (left). Dye is injected via the distal lumen into the aortic root, visualizing the coronary arteries and competent aortic valve. Intraoperative TEE of the aortic root (long-axis view 130 degrees) showing the position of the endovascular aortic clamp at a distance of $2.6 \mathrm{~cm}$ from the aortic anulus (right).

CFM, Sonotron, Norway) (Fig. 3) in the first 20 patients. Later in the series, placement of the clamp was guided by TEE only. ${ }^{6}$ The endovascular aortic clamp was inflated to endoluminally block the ascending aorta while the heart was vented through the endovascular pulmonary vent and the distal lumen of the endovascular aortic clamp in the aortic root. After clamping, balloon pressure was continuously measured and maintained between 250 and 340 $\mathrm{mm} \mathrm{Hg}$. Additional volume was inflated in case of a decrease in balloon pressure below $220 \mathrm{~mm} \mathrm{Hg}$. Cold crystalloid cardioplegic solution (Bretschneider HTK solution, Köhler Chemie, Alsbad, Germany) (2600 \pm 950 $\mathrm{ml}$ ) was delivered antegradely through the distal endovascular aortic clamp lumen while maintaining aortic root pressure between 50 and $70 \mathrm{~mm} \mathrm{Hg}$. After cardiac arrest had been established, the left atrium was opened and the mitral valve exposed by a specially designed atrial retractor (Heartport) that was inserted through a second $5 \mathrm{~mm}$ port at the sixth or seventh right intercostal space parasternally. Mitral valve repair or replacement was performed under three-dimensional videoscopic guidance with the use of specially designed instruments (Heartport). After completion of the procedure the left atrium was closed by a continuous suture. Deairing was performed by inflation of the lungs and simultaneous reduction of venous drainage with the patient placed in the Trendelenburg position. Deairing of the aortic root was performed by suction via the distal lumen of the endovascular aortic clamp. The endovascular aortic clamp was deflated and the catheter was left in place for further venting until deairing was completed. If necessary, defibrillation was performed via external defibrillation pads. A temporary pacing wire (Biotronik, Berlin, Germany) was placed in the pericardial sac. The endovascular aortic clamp was then withdrawn and the chest wound closed. A drainage tube was placed through the access port for the stereoscope. After appropriate reperfusion, the arterial and venous cannulas were removed and the femoral vessels reconstructed.

Follow-up. Pain assessment during hospital stay and follow-up was performed with the use of a visual analog scale $(0$ to $10 \mathrm{~cm})$ ranging from no pain (0) to most intense pain (10). The patients were asked to mark the scale corresponding to their pain perception. Preoperative and postoperative activity level was judged by the Duke activity status index, a 12-item questionnaire that assesses physical functioning. ${ }^{7}$ Follow-up examinations at 6 and 12 weeks included a physical examination, chest radiograph, electrocardiogram, transthoracic echocardiogram, and spirometric examination. Data are presented as median and range or mean \pm standard deviation. Adverse events are reported according to the guidelines for reporting morbidity and mortality after cardiac valvular operations. ${ }^{8}$

\section{Results}

Catheter placement. Positioning of the endovascular pulmonary vent was possible in all patients. Cannulation in the groin was performed without complication in all but one patient, in whom the venous cannula could not be advanced transfemorally into the inferior vena cava. In this patient the incision was enlarged and the right atrium was cannulated directly. Placement and positioning of the endovascular aortic clamp was uneventful in all but one patient. The tip of the balloon of the 
Table II. Types of procedures for which the PortAccess system was used

\begin{tabular}{lr}
\hline \multicolumn{1}{c}{ Type of procedure } & $n$ \\
\hline Quadrangular resection and ring implantation & 11 \\
Isolated ring implantation & 11 \\
Isolated commissurotomy & 1 \\
Plication plasty and chordal replacement & 2 \\
Commissurotomy and ring implantation & 2 \\
Commissurotomy and chordae replacement & 1 \\
Sliding plasty & 2 \\
Mitral valve replacement & 23 \\
\hline
\end{tabular}

endovascular aortic clamp was placed at a distance of 2 to $4 \mathrm{~cm}$ from the aortic valve anulus (mean $2.8 \pm 0.6 \mathrm{~cm}$ ) (Fig. 3). In all patients, injection of an initial volume of 20 to $35 \mathrm{ml}$ (mean inflation volume of $24 \pm 6 \mathrm{ml}$ ) resulted in sufficient aortic occlusion with a balloon pressure in the desired range of 235 to $360 \mathrm{~mm} \mathrm{Hg}$. Larger volumes were observed in patients with a large aorta. In six patients, additional volumes had to be added because the balloon pressure decreased below $220 \mathrm{~mm} \mathrm{Hg}$ during the procedure. Migration of the balloon during initial placement was observed in six patients but was easily corrected by external manipulation of the catheter under TEE control. In one patient the balloon migrated into the left ventricle in the middle of the procedure, causing severe transaortic regurgitation. Repeat dye injection after retraction of the balloon confirmed severe aortic insufficiency. In this patient, the operation was continued with the heart fibrillating. At the end of the procedure, aortic valve function had completely recovered as demonstrated by TEE. Balloon rupture was observed in another patient and was noticed by the onset of ventricular fibrillation. The operation was again completed with the use of hypothermic fibrillatory arrest.

Surgical technique. In all patients, the mitral valve was accessible through the right lateral minithoracotomy. The mean length of the incision was $5.4 \pm 1.8 \mathrm{~cm}$ (range 3.8 to $8 \mathrm{~cm}$ ) with the last 20 patients having incisions of $4.5 \mathrm{~cm}$ or less. In the first eight patients a partial resection of the fifth rib was performed. In the last 43 patients, rib resection could be avoided by using our custom-made rib retractor. In one patient, insertion of the left atrial retractor through the sixth intercostal space resulted in injury of the distal right internal thoracic artery, which necessitated its ligation.

In 28 patients, the mitral valve was repaired under direct and stereoscopic vision by a combination of repair techniques including quadrangular resection, commissurotomy, sliding plasty, chordal replacement, and annuloplasty with partial (Cosgrove ring, Baxter Health Care Corporation, Santa Ana, Calif.) or complete (Séguin ring, St. Jude Medical, Inc., St. Paul, Minn., or Physio ring, Baxter) mitral valve rings (Table II). As demonstrated by intraoperative TEE, successful repair was achieved in all but three patients. One patient had new moderate mitral stenosis (mitral valve orifice area $1.6 \mathrm{~cm}^{2}$ ) after a complex repair of a calcified valve including quadrangular resection of the posterior leaflet. Two patients had residual grade II mitral insufficiency. In these patients, the mitral valve was replaced in the same session by means of the same access and surgical technique. Twenty-three patients (20 initial operations and three after failed repair) underwent mitral valve replacement with preservation of the posterior mitral valve leaflet. Additional preservation of part of the anterior leaflet with resuspension of its chordae was performed in seven patients. We preferably use the rotatable St. Jude Medical mitral valve (Master Series) because of its relatively soft cuff, which allows for safer knot tying. Mean duration of operation, cardiopulmonary bypass, and crossclamp times were $196 \pm 53,133 \pm 52$, and $72 \pm$ 27 minutes, respectively. In 37 patients, a spontaneous rhythm was present after the endovascular aortic clamp was deflated, whereas 14 patients required one or more external defibrillations of 200 to 360 joules without evidence of skin irritation. In six patients conversion to an enlarged thoracotomy incision $(n=2)$, a ministernotomy $(n=2)$, or complete sternotomy $(n=2)$ was necessary. Acute retrograde aortic dissection occurred in two patients. In one patient, TEE revealed a rapidly progressing dissection to the level of the aortic valve with the onset of retrograde extracorporeal perfusion. Emergency median sternotomy and supracoronary replacement of the ascending aorta with reconstruction of the aortic arch was performed with a circulatory arrest time of 22 minutes. The mitral valve was replaced conventionally. After a prolonged recovery, the patient was finally transferred to a neurologic rehabilitation center. In another patient aortic dissection became evident during weaning from cardiopulmonary bypass. The patient was converted to a median sternotomy and had replacement of the ascending aorta but finally died of persistent low cardiac output.

Postoperative course and complications. Primary median intubation time was 25.5 hours (range 5 to 264 hours), with seven patients requiring ventilation 


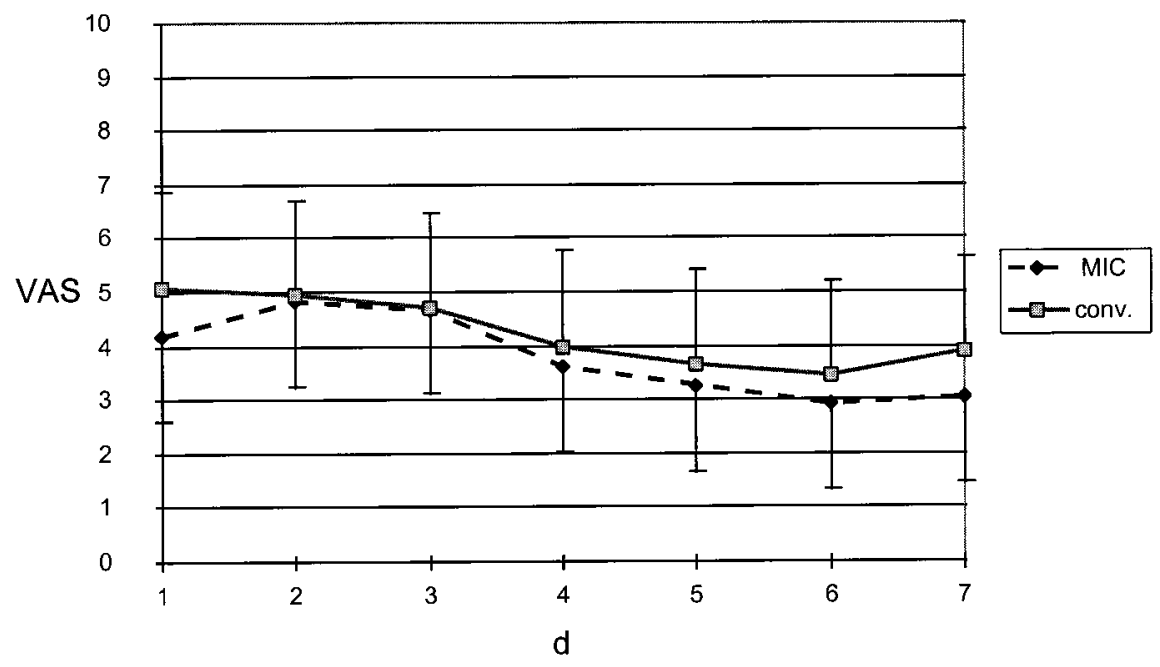

Fig. 4. Day by day pain perception graded by visual analog scale (VAS). Comparison of 20 patients undergoing conventional mitral valve surgery (conv.) as compared with 20 patients who underwent the minimally invasive procedure (MIC). Error bars indicate standard error of the mean. $d$, Days.

for more than 48 hours. Duration of intensive care and hospital stay was 2 days (range 1 to 36 days) and 13 days (10 to 36 days), respectively. In most patients pain medication could be stopped on the second postoperative day. However, when they were compared with a group of 20 patients who had conventional mitral valve surgery via a median sternotomy, no significant difference in pain perception was apparent (Fig. 4). Median blood loss was $800 \mathrm{ml}$ (range 175 to $2130 \mathrm{ml}$ ) with three patients requiring reexploration for bleeding without enlarging the primary incision. One patient was bleeding from the videoscope port site and two had bleeding from intercostal arteries after partial rib resection. Intraoperative and postoperative complications are summarized in Table III.

Five patients $(9.8 \%)$ died in the perioperative period or at follow-up. One patient with long-term oral cortisone therapy died on postoperative day 12 after he had been referred to a specialized center for treatment of toxic epidermal necrolysis that developed as a result of a superficial skin infection. One patient who had successful mitral valve repair had acute onset of recurrent mitral insufficiency on the third postoperative day because the mitral ring had torn free. Mitral valve replacement was performed subsequently but the patient died of irreversible low cardiac output. One patient died of pneumonia on the sixth postoperative day after uneventful recovery. One patient died of pulmonary embolism on postoperative day 24 despite adequate anticoagulation.

At discharge after mitral valve repair, all patients had normal mitral valve function with no or only trivial regurgitation. All implanted valves were functioning normally, as shown by postoperative echocardiographic studies. At a mean follow-up of $261 \pm$ 13 days, all patients except two had at least returned to their preoperative activity level. Baseline mean Duke activity index score was $19.3 \pm 11.3$ before the operation and increased to $23.2 \pm 10$ at 6 weeks' and $24.2 \pm 10.3$ at 12 weeks' follow-up, respectively. New York Heart Association class had improved at least by one class in all but six patients. The patient with phrenic nerve palsy was still limited by tachypnea and had reduced vital capacity. In all other patients, respiratory function had normalized.

Three patients who had mitral valve replacement and normal mitral valve function at their pre-discharge echocardiographic examination were readmitted with paravalvular leakage after 5, 7, and 16 weeks, respectively. One patient was transferred shortly before her routine 6-week outpatient follow-up examination with acute onset of dyspnea. This patient underwent a successful reoperation using a conventional approach via a median sternotomy. At the time of reoperation, one of the sutures that had been placed through the heavily calcified posterior segment of the mitral valve anulus was found to have torn. In one asymptomatic patient, 
Table III. Perioperative complications

\begin{tabular}{|c|c|c|}
\hline Complications & Cause & $n$ \\
\hline \multicolumn{3}{|l|}{ Cardiac } \\
\hline Prolonged catecholamine support ( $<3$ days) & & 9 \\
\hline \multirow{2}{*}{ Rhythm disorders } & Supraventricular tachycardia & 8 \\
\hline & Intermittent AV-block & 2 \\
\hline \multicolumn{3}{|l|}{ Bleeding } \\
\hline \multirow[t]{3}{*}{ Reexploration for bleeding } & Source: Intercostal artery & 2 \\
\hline & Port site & 1 \\
\hline & Lung adhesions & 1 \\
\hline \multicolumn{3}{|l|}{ Pulmonary } \\
\hline \multirow[t]{2}{*}{ Reintubation for respiratory insufficiency } & Right phrenic nerve palsy & 1 \\
\hline & Atelectasis, pneumonia & 3 \\
\hline Prolonged ventilation $>48 \mathrm{hr}$ & Atelectasis, pneumonia & 4 \\
\hline Pneumothorax, skin emphysema & & 5 \\
\hline Pleural effusion & & 5 \\
\hline \multicolumn{3}{|l|}{ Neurologic } \\
\hline Postoperative confusion & Embolism (?) & 9 \\
\hline Hemiplegia (transient) & Aortic dissection, balloon displacement (?), embolism (?) & 4 \\
\hline \multicolumn{3}{|l|}{ Vascular/groin } \\
\hline Lymph fistula & & 1 \\
\hline Delayed wound healing & & 1 \\
\hline \multicolumn{3}{|l|}{ Other } \\
\hline Aortic dissection & Perforation of aortic wall & 2 \\
\hline Renal failure (CVVH) & Low cardiac output & 2 \\
\hline Pancreatitis & Unknown & 1 \\
\hline Upper GI bleed & Stress ulceration & 1 \\
\hline Toxic skin necrolysis & Unknown & 1 \\
\hline
\end{tabular}

CVVH, Continuous veno-venous hemofiltration; GI, gastrointestinal.

non-pledget-supported sutures had been used at the primary operation. One patient with symptomatic paravalvular leakage had ongoing endocarditis at the time of the primary operation. At reoperation, the knots appeared to be tight, but some sutures had torn through the mitral valve anulus. Both patients underwent successful reoperations performed through a median sternotomy. Remarkably, in all patients there were almost no adhesions at reoperation. In all except three patients, wound healing at the groin was uncomplicated. Two patients had a small residual hematoma and one patient had a lymph fistula in the right groin, all of which were treated conservatively. However, at 12 weeks' follow-up, three patients had increased flow (>3 $\mathrm{m} / \mathrm{sec}$ ) at the femoral artery as demonstrated by Doppler studies, indicating mild stenosis at the level of cannulation.

\section{Discussion}

Minimally invasive procedures were introduced in expectation of reducing postoperative pain, recovery time, and cost while providing a favorable cosmetic result as compared with conventional surgery. With the use of the Port-Access system, mitral valve surgery including complex repair procedures through an incision less than $4 \mathrm{~cm}$ has become possible. The three-dimensional view provided by the stereoscope facilitates repair procedures because it provides a detailed visualization of all valve structures.

With increasing experience, cardiopulmonary bypass and crossclamp times became shorter in this series. The length of the thoracotomy incision has steadily decreased, and partial rib resection is no longer performed. However, serious complications occurred. Two retrograde aortic dissections were of major concern. Both events were most likely caused by intimal dissection at the level of the iliac artery induced by the guide wire. Retrograde flow led to complete retrograde aortic dissection. Because of the limited experience with the Port-Access system, no conclusions can be drawn to determine the relative risk for the described severe complication. However, as of May 1997, the Heartport World Wide Registry reported aortic dissection in 11 of 610 patients undergoing Port-Access surgery, ${ }^{9}$ a number that clearly exceeds the incidence of aortic dissection after conventional cardiac operations. ${ }^{10} \mathrm{Al}-$ though only one death was clearly procedure re- 
lated, the mortality associated with the procedure in this single-center experience exceeded our own and the reported mortality for conventional surgery for nonischemic mitral valve insufficiency or mitral stenosis. ${ }^{11}$ In addition, there was considerable morbidity not allowing for a reduction of mean hospitalization time. The duration of intubation could not be reduced as compared with standard mitral valve repair or replacement. This might be explained by the use of single-lung ventilation in the beginning of the series, resulting in residual atelectasis during recovery. We therefore abandoned double-lumen intubation and instead attempt to maintain a minimum of ventilation to both lungs. With increasing experience, bleeding problems became less frequent as potential bleeding sites were identified and eliminated by careful videoscopic inspection of port sites and by abandoning rib resection. A problem that was infrequently encountered early in this series was incomplete deairing of the heart, which might partially explain the high incidence of postoperative confusion. Enhancement of the venting capacity of the endovascular aortic clamp might further reduce this problem. Surgical manipulation of the valve and the perivalvular tissue is limited by a decreased range of movement and the use of longer instruments with limited stability. Especially in the presence of annular calcification, placement of sutures can be difficult. Knotting is performed outside the chest and knots are placed with the use of a knotpusher. This is a time-consuming and relatively insecure procedure, which prolongs aortic crossclamp time by 10 to 15 minutes. Refinement of the currently used instruments is therefore highly desirable.

Manipulation of an atherosclerotic aorta (i.e., aortic clamping, cannula insertion) may result in atheroembolism causing neurologic damage. ${ }^{12-14}$ Although some investigators believe that internal balloon occlusion of the aorta is a safe procedure, which is advantageous in patients with a calcified aorta, ${ }^{15}$ it is as yet not known if an internal clamping pressure of up to $360 \mathrm{~mm} \mathrm{Hg}$ applied for as long as 2 hours is of no harm. In one patient who required conversion for bleeding, we noticed a subadventitial hemorrhage of the ascending aorta. With TEE, aortic dissection was excluded so that surgical intervention was not required. The tendency of the balloon to migrate requires continuous observation. Proximal displacement may, in theory, damage the aortic valve, and distal migration may obstruct the brachiocephalic trunk. We observed transient hemiparesis in four patients, a possible cause being temporary translocation of the balloon. There was no change in right radial artery pressure, so that monitoring of right radial artery pressure alone cannot be considered reliable for exclusion of balloon displacement. Once the left atrium is opened, TEE also fails to locate the balloon properly. Clearly a method to continuously monitor balloon position and carotid artery flow should be introduced. In the last 10 cases we applied transcranial Doppler measurements to detect emboli and to continuously monitor blood flow in the middle cerebral arteries. With femoral cannulation and retrograde aortic flow, there is the potential disadvantage of flushing debris of atheromatous plaques originating from the abdominal or thoracic aorta into the cerebral circulation. It is therefore mandatory to examine the aorta with TEE before placement of the endovascular aortic clamp to identify patients with atheromatous aortic plaques or thrombi and to exclude these patients from an approach that requires retrograde perfusion. In addition, femoral cannulation may cause local complications at the groin, as well as ischemic complications of the leg. ${ }^{16}$ Careful patient selection excluding patients with severe atherosclerosis, kinking, or ectasia of the aorta and the peripheral arteries is therefore advisable.

The term "minimally invasive" has been applied to a number of different techniques for mitral valve procedures, ${ }^{17-19}$ but there is still no agreement on what type of procedure should be called minimally invasive. ${ }^{20}$ Navia and Cosgrove ${ }^{21}$ reported on 25 patients who had a $10 \mathrm{~cm}$ right parasternal incision with resection of the third and fourth cartilages and sacrifice of the right internal thoracic artery. Unfortunately, these authors provided no data to support the conclusion that the smaller incision resulted in a reduction of patient discomfort, a reduction in the incidence and risks of wound infection and blood loss, and a reduction of recovery time and costs. For these questions to be appropriately answered, controlled randomized studies enrolling larger series of patients may be necessary. In several patients (including those having reoperations) we have performed minimally invasive mitral valve repair with the use of hypothermic fibrillatory arrest, as initially suggested by Lin and associates. ${ }^{22}$ A different approach for less invasive mitral valve replacement via a midaxillary thoracotomy, applying femoro-femoral bypass and direct transcutaneous aortic clamping with a modified aortic clamp inserted through an additional posterior axillary line incision, has been suggested by Chitwood and colleagues. ${ }^{23}$ Although 
the incision is not as small as with the Port-Access procedure, this approach seems to provide safe antegrade cardioplegic cardiac arrest. Both techniques avoid the use of additional catheters that clearly increase the overall costs of the procedure. Our initial experience applying this technique in eight patients is very satisfying.

In conclusion, the results of our preliminary study with the video-assisted Port-Access technique have to be judged with caution because a number of serious complications developed. In the last 28 patients who have been operated on since this manuscript was submitted, the morbidity was low and no deaths occurred. Average ischemic time could be further reduced to less than 50 minutes. Clearly, the design and instrumentation of the PortAccess system needs further improvement, including an antegrade perfusion system to enhance the safety and the efficacy of the approach. The current technique should be restricted to well-trained surgeons with a high volume of cases, so that they can gain sufficient experience with this novel procedure.

We thank Mario Pompili, MD, Thomas Burdon, MD, and Lawrence C. Siegel, MD, from the Stanford School of Medicine, for their assistance in the animal studies that preceded the clinical trial presented here, as well as for their help and expertise in the first clinical cases. We also thank Miss Monika Friedrich for her help in preparing the manuscript.

\section{REFERENCES}

1. Stevens JH, Burdon TA, Peters WS, Siegel LC, Pompili MF, Vierra MA, et al. Port-access coronary artery bypass grafting: a proposed surgical method. J Thorac Cardiovasc Surg 1996;111:567-73.

2. Schwartz DS, Ribakove GH, Grossi EA, Stevens JH, Siegel LC, St Goar FG, et al. Minimally invasive cardiopulmonary bypass with cardioplegic arrest: a closed chest technique with equivalent myocardial protection. J Thorac Cardiovasc Surg 1996;111:556-66.

3. Stevens JH, Burdon TA, Siegel LC, Peters WS, Pompili MF, St Goar FG, et al. Port-access coronary artery bypass with cardioplegic arrest: acute and chronic canine studies. Ann Thorac Surg 1996;62:435-41.

4. Pompili MF, Stevens JH, Burdon TA, Siegel LC, Peters WS, Ribakove GH, et al. Port-access mitral valve replacement in dogs. J Thorac Cardiovasc Surg 1996;112:1268-74.

5. Reichenspurner H, Guilielmos V, Daniel WG, Schüler S. Minimally invasive coronary artery bypass surgery [letter]. N Engl J Med 1997;336:67-8.

6. Falk V, Walther T, Diegeler A, Autschbach R, Wendler R, van Son JAM, et al. Echocardiographic monitoring of minimally invasive mitral valve surgery using an endoaortic clamp. J Heart Valve Dis 1996;5:630-7.

7. Hlatky MA, Boineau RE, Higginbotham MB, Lee KL, Mark DB, Califf RM, et al. A brief self-administered questionnaire to determine functional capacity (the Duke Activity Status index). Am J Cardiol 1989;64:651-4.

8. Edmunds LH, Clark RE, Cohn LH, Grunkemeier GL, Miller C, Weisel RD. Guidelines for reporting morbidity and mortality after cardiac valve operations. Ann Thorac Surg 1996; 62:932-5.

9. Reitz B. Presentation at the World Congress on Minimally Invasive Cardiac Surgery. Paris. May 1997.

10. Still RJ, Hildenberg AD, Akins CW. Intraoperative aortic dissection. Ann Thorac Surg 1992;53:374-80.

11. Ferrazzi P, McGiffin DC, Kirklin JW, Blackstone EH, Bourge RC. Have the results of mitral valve replacement improved? J Thorac Cardiovasc Surg 1986;92:186-97.

12. Mills SA. Risk factors for cerebral injury and cardiac surgery. Ann Thorac Surg 1995;59:1296-9.

13. Blauth CI. Macroemboli and microemboli during cardiopulmonary bypass. Ann Thorac Surg 1995;59:1300-3.

14. Barbut D, Hinton RB, Szatrowski TP, Hartman GS, Bruefach M, Williams Russo P, et al. Cerebral emboli detected during bypass surgery are associated with clamp removal. Stroke 1994;25:2398-402.

15. Haenen I, Rodrigus I, Boeckxstaens, Amsel BJ, Moulijn AC. Intermittent internal balloon occlusion: a safe and simple technique for clamping heavily calcified aortas [abstract]. J Cardiovasc Surg 1996:4(Suppl I):166.

16. Gates JD, Bichell DP, Rizzo RJ, Couper GS, Donaldson MC. Tight ischemia complicating femoral vessel cannulation for cardiopulmonary bypass. Ann Thorac Surg 1996;61:730-3.

17. Carpentier A, Loulmet D, Carpentier A, Le Bret E, Haugades $\mathrm{B}$, Dassier $\mathrm{P}$, et al. Chirurgie à coeur overt par vidéo-chirurgie et mini-thoracotomie. Premier cas (valvuloplastie mitrale) opéré avec succèss. CR Acad Sci III 1996; 319:219-23.

18. Benetti FJ, Rizzardi JL, Pre L, Polanco A. Mitral valve replacement under video assistance through a minithoracotomy. Ann Thorac Surg 1997;63:1150-2.

19. Arom KV, Emery RW. Minimally invasive mitral operations [letter]. Ann Thorac Surg 1997;63:1219-20.

20. Lytle BW. Minimally invasive cardiac surgery. J Thorac Cardiovasc Surg 1996;111:554-5.

21. Navia JL, Cosgrove DM. Minimally invasive mitral valve operations. Ann Thorac Surg 1996;62:1542-4.

22. Lin PJ, Chang CH, Chu JJ, Liu HP, Tsai FC, Chu PH. Video assisted mitral valve operations. Ann Thorac Surg 1996;61: 1781-7.

23. Chitwood WR, Elbeery JR, Chapman WHH, Moran JM, Lust RL, Wooden WA. Video assisted minimally invasive mitral valve surgery: The "micro-mitral" operation. J Thorac Cardiovasc Surg 1997;113:413-4.

\section{Discussion}

Dr. W. Randolph Chitwood, Jr. (Greenville, N.C.). Dr. Mohr has presented among the first series of patients undergoing video-assisted minimally invasive mitral valve surgery. He and his colleagues are to be applauded for their careful and critical evaluation of their results. He has emphasized that these techniques are in evolution, with potentially a steep learning curve. In this series a right anterolateral minithoracotomy was used. Centrifugal pump-assisted venous drainage was used in conjunction with retrograde femoral arterial perfusion. Complex mitral valve repairs were done in 29 patients with good 
results. Three-dimensional videoscopy was very helpful in these patients. Postoperative pain indices were low and activity scores high. However, intubation and intensive care times were not reduced compared with other mitral valve operations. Moreover, the operative mortality was $9.8 \%$. For comparison, the Heartport method has provided a $6.3 \%$ mortality for the over 250 patients having mitral operations worldwide to date.

In Dr. Mohr's series, technique-related complications were significant. In two instances the intraaortic balloon clamp either migrated into the ventricle or ruptured. In five patients a transient hemiparesis developed, presumably as a result of proximal balloon displacement. There were two cases of retrograde aortic dissection.

Recently our group at East Carolina University has performed 31 video-assisted mitral valve operations using a minithoracotomy and direct aortic occlusion with a new percutaneous transthoracic aortic clamp. Our results at present suggest lower cost, shorter hospital stays, and a $3.2 \%$ mortality. Moreover, we had no neurologic complications and no aortic injuries. We believe that the clamp method may be more secure, safer, and more economical than balloon occlusion.

I have three questions for Dr. Mohr. In this initial series, how have you selected and screened your patients before the operation? From what you have learned already, how can neurologic events be avoided? How do you think direct aortic occlusion may compare in the long run with these more expensive balloon occlusive devices?

We agree with Dr. Mohr that video assistance can facilitate portions of these operations quite well. However, the development of intracardiac video-dexterity requires a learning curve. Using standard two-dimensional thoracoscopic vision, we have found chordal transfers and quadrangular resections difficult without direct vision, but they can be done.

Dr. Mohr, please give us your thoughts regarding the Zeiss three-dimensional stereoscope used in your cases. Also, if you can, compare this with the other commercial three-dimensional visioning devices available.

This paper has provided important new information regarding these evolving techniques. However, we must remain circumspect regarding operative safety and valve durability until long-term results are delivered that compare with results of conventional operations.

Dr. Eugene Grossi (New York, N.Y.). At New York University we have performed 100 minimally invasive procedures in which the endovascular aortic clamp was used and with a complication rate that is less than in your report. This raises some concerns relating to your technique. First, did you meticulously adhere to Seldinger guide-wire techniques? We have not seen dissection when these principles were adhered to. In which patients did you intraoperatively abandon the attempt to use retrograde perfusion? Finally, with regard to neurologic complications, what are your deairing guidelines? These technical issues are very important as we learn this new technology.

Dr. Alain F. Carpentier (Paris, France). I rise to congratulate Dr. Mohr for bringing to our attention the potential complications linked with any type of videoassisted minimally invasive surgery. Starting as early as
February 1996, we have used different techniques of video-assisted minimally invasive surgery, including the Heartport system. We have 22 mitral valve reconstructions with no deaths and only one case of reoperation.

Using the Heartport system, we found that it is possible to place a balloon with TEE without requiring fluoroscopy. You mentioned TEE. Would you now avoid using fluoroscopy? Second, on the basis of your experience, what are your current indications and contraindications for minimally invasive surgery and for the Heartport system? Third, we have been somewhat disappointed by the pain after this operation when using thoracotomy. How do you manage this problem, since one of the potential advantages of minimally invasive techniques is to reduce disability and pain for the patient.

Dr. Mohr. We have used an independent investigator to evaluate pain in all of our 250 patients who underwent minimally invasive surgery. As yet, the results are very disappointing. Among our German patients pain perception is very similar. Maybe it is different in the United States. We could not demonstrate significant differences, neither for our 115 patients who had coronary bypass surgery nor for our 50 patients having aortic valve replacement via small incisions.

Concerning the other remarks, during the time encompassed by this study, 250 patients underwent conventional mitral valve surgery. In the 50 patients we followed a protocol that was part of a multicenter study which regulated the selection of patients. Patients who were older than 75 years, having concomitant cardiac disease, and having reoperations were not included in the study. We selected the patients carefully. However, some of them had pulmonary hypertension as a consequence of severe long-lasting mitral valve disease. We also performed 30 other approaches. We followed Professor Carpentier's, Professor Cosgrove's, and also Professor Chitwood's techniques. We tried to exclude very obese patients for the Heartport technique because the distance from the incision sometimes exceeded the length of the instruments.

I mentioned that we also used clamping techniques. I have used Dr. Chitwood's technique with direct aortic clamping in eight patients so far. The only concern is that if you operate through an incision as small as $4 \mathrm{~cm}$, and you place the clamp through a port, in my eyes it is still difficult to safely place the clamp at the ascending aorta and not injure the pulmonary artery. You can solve this problem if you go for a larger incision, more anteriorly. The reason we chose the lateral incision is for optimal view of the valve, which is then directly in view.

We tried several two-dimensional and three-dimensional videoscopic systems. However, if you use the very small incisions, the three-dimensional system is extremely helpful, especially in complex repairs. For example, it is almost impossible to adjust the length of the chordae with a polytetrafluoroethylene suture using a two-dimensional videoscopic system; the three-dimensional system is also advantageous to estimate the coaptation of the valves after repair. I did not have the chance to work with the Vista System (VISTA Cardiothoracic Surgery, Westborough, Mass.), which is available in the United States. However, I am uneasy about having so much electronic 
equipment on my head. At least on the European market the Zeiss three-dimensional system was, in our eyes, a superior system.

Now I would like to answer Dr. Grossi's question. Of course we use guide wires. I think the guide wire supplied with this system is very stiff and may create an intimal tear at the site of the iliac arteries. We had the unfortunate opportunity to do an autopsy on one of our patients who died because of early dissection. The reason for the retrograde dissection was an intimal tear at the site of the common iliac artery.

If you look at the history of our two dissections, the guide wire was advanced into the ascending aorta, and so was the balloon, under guidance in the first case with fluoroscopy and echo. Nothing happened at the time of insertion, but rather when we started retrograde perfusion. The retrograde dissection expanded from the abdominal to the descending and ascending aorta. I think the underlying mechanisms are intimal tears that lead to dissections with retrograde perfusion. We have to find a way to perfuse the descending aorta that might solve the problem. I know Heartport is working on this very intensively and I am looking forward to a new device.

In my eyes, deairing is still a problem because every- body is used to a needle vent in the ascending aorta at the roof of the ascending aorta. This is not possible with the current Port-Access approach. At the beginning, we did see ST elevation in almost all patients when we released the balloon clamp. We changed our protocol in the last 30 patients, and as soon as the valve is replaced or repaired, we reperfuse the aortic root with blood, with the balloon still inflated. We position the patient very much to the left side, cannulate the left ventricle through the mitral valve, and then close the left atrium while the heart is already beating. Nevertheless, sometimes air is continuously released when the heart is already beating and the venting capacity of the endovascular clamp is not effective.

We never use retrograde cardioplegia in our patients. If we have a prolonged crossclamp time, we repeat antegrade cardioplegia through the endovascular clamp. I am reluctant to use a retrograde percutaneous catheter because of the risk of injuring the coronary sinus. The sinus cannot be reached through this small incision.

Professor Carpentier, we abandoned fluoroscopy. Our residents, like Dr. Falk, are very experienced in guiding the endovascular aortic clamp placement, the guide wire, through the descending aorta, through the arch, to its final position in the ascending aorta, and using fluoroscopy is a nuisance. 